Research Paper

\title{
Chuanhu Anti-Gout Mixture versus Colchicine for Acute Gouty Arthritis: A Randomized, Double-Blind, Double-Dummy, Non-Inferiority Trial
}

YanGang Wang1, Luan Wang1, EnZe Li², Yang Li ${ }^{1}$, ZhongChao Wang ${ }^{1}$, XiaoFang Sun ${ }^{1}$, XiaoLong Yu ${ }^{1}$, Lin $\mathrm{Ma}^{2}$, YunLong Wang ${ }^{\circledR}$, YouXin Wang ${ }^{\circledR}$

1. Department of endocrinology, The Affiliated Hospital of Medical College Qingdao University, 16 Jiangsu Road, Qingdao, 266003, China.

2. Department of pharmacy, The Affiliated Hospital of Medical College Qingdao University, 16 Jiangsu Road, Qingdao, 266003, China.

3. Gout laboratory, The Affiliated Hospital of Medical College Qingdao University, Shandong Provincial Key Laboratory of Metabolic Diseases, Qingdao, 266003, China.

4. Beijing Municipal Key Laboratory of Clinical Epidemiology, School of Public Health, Capital Medical University, Beijing 100069, China.

$\triangle$ Corresponding author: A/Professor Youxin Wang, Beijing Municipal Key Laboratory of Clinical Epidemiology, School of Public Health, Capital Medical University, Beijing 100069, China; Tel: 008610 83911497; E-mail: wangy@ccmu.edu.cn Dr. Yunlong Wang, Gout laboratory, The Affiliated Hospital of Medical College Qingdao University. Shandong Provincial Key Laboratory of Metabolic Diseases. Tel: 0086-15092269969; E-mail: wylapollo@163.com.

() Ivyspring International Publisher. This is an open-access article distributed under the terms of the Creative Commons License (http://creativecommons.org/ licenses/by-nc-nd/3.0/). Reproduction is permitted for personal, noncommercial use, provided that the article is in whole, unmodified, and properly cited.

Received: 2014.03.20; Accepted: 2014.06.0I; Published: 2014.06.14

\begin{abstract}
Background The Chuanhu anti-gout mixture has been used for many years in the treatment of gout in Chinese Traditional Medicine, and current methods for treatments for acute gouty arthritis have been either less effective or have had serious side effects.

Methods In this I2-week, double-blind, double-dummy, non-inferiority study, outpatient individuals with newly diagnosed acute gouty arthritis were randomly assigned to receive Chuanhu anti-gout mixture or colchicine. Both the study investigators and the participants were masked to the treatment assignments. The primary outcome was the recurrence rate of acute gouty arthritis, and the secondary outcomes were changes in white blood cells (WHC) and C-reactive protein (CRP). This trial is registered at ISRCTN.org as trial ISRCTN6521994I.

Results A total of 176 patients were randomly assigned to receive either the Chuanhu anti-gout mixture or Colchicine. The overall recurrence rates in the Chuanhu anti-gout mixture group $(\mathrm{CH}$ group) and the Colchicine group (Col group) were $12.50 \%$ vs $14.77 \%$ (difference $-2.22 \%$, $95 \%$ confidence interval $(95 \% \mathrm{Cl}):-10.78 \% \sim 6.23 \%)$, meeting the predefined non-inferiority criterion of $15 \%$, as did the data for WHC and CRP.The incidence of adverse events (mainly diarrhea) was less in the Col group than in the $\mathrm{CH}$ group $(2.27 \%$ vs $28.41 \%, 95 \% \mathrm{Cl} 0.01 \sim 0.26)$. In addition, changes in blood uric acid, alanine aminotransferase, aspartate aminotransferase and creatinine in the $\mathrm{CH}$ group were significantly larger compared to those in the Col group $(P<0.05)$.

Conclusions The Chuanhu anti-gout mixture was non-inferior to colchicine for the treatment of acute gouty arthritis. The study suggested that the Chuanhu anti-gout mixture can be considered an alternative choice for the treatment of acute gouty arthritis because of its lower incidence of adverse events and its protection of kidney and renal function.
\end{abstract}

Key words: Chuanhu, acute gouty arthritis

\section{Introduction}

Acute gout is an intensely painful, inflammatory arthritis with a rapidly escalating inflammatory response resulting from the formation of monosodium urate crystals in the affected joint space [1]. It is the most common form of inflammatory joint disease in men over the age of 40 years [2]. Correspondingly, the 
prevalence and incidence of gout have been increasing, and gout is currently one of the most common causes of inflammatory arthritis in most industrialized countries [3]. Epidemiological surveys have indicated that the gout incidence rate was $1.14 \%$ in the coastal cities of Shandong province in China [4] and $1.4 \%$ in Europe [5]. One acute attack of gout may be the only manifestation, or acute inflammatory arthritis episodes (flares), often monoarticular, may recur [6]. If untreated, these flares may lead to chronic gouty arthropathy and deposition of urate crystals in soft tissues, forming tophi [6]. It has been reported that hyperuricemia and gout could not only cause joint swelling, but they could also induce and aggravate dyslipidemia, diabetes and cardiovascular disease, resulting in increased mortality in patients with cardiovascular and cerebrovascular diseases, metabolic syndrome or kidney disease [7-9].

Acute gouty arthritis is characterized by intensely painful, inflammatory arthritis with a rapidly escalating inflammatory response resulting from the formation of monosodium urate crystals in the affected joint space [10]. Current standard treatments for acute gouty arthritis include non-steroidal anti-inflammatory drugs (NSAIDs), colchicine and corticosteroids [11]. Some patients respond poorly to these therapies, and/or they cannot be safely utilized due to co-morbid medical conditions [11].

Chinese herbal compounds with high efficacy and a low incidence of adverse reactions have drawn increasing attention from scholars. Based on years of clinical practice that considered the Traditional Chinese Medicine pathogenesis of gout, the Chuanhu anti-gout mixture was invented. It is composed of 9 herbs, including Caulis Lonicerae, Rhizoma Polygoni Cuspidati, and Discorea nipponica Makino. Since 1999, this Chinese medicine compound has been used to treat more than 600 gout cases with high efficacy and safety. It has been well received by patients and was accepted for a national invention patent in 2000. The Chuanhu anti-gout mixture might be effective for therapy of acute gouty arthritis and might have less side effects compared to colchicine. The possible risks to participants included the adverse reactions of pain, swelling, and reduced joint mobility, together with injury to the liver and kidney.

In order to test its efficacy and monitor its adverse effects, we performed a randomized controlled trial in Qingdao, Shandong Province, China.

\section{Materials and Methods}

\section{Patients}

Inclusion criteria: Adults (18 years and older) with a new clinical diagnosis of gout according to the 1977 American College of Rheumatology classifica- tion criteria and an onset of the disease duration of less than 48 hours were eligible to participate.

Exclusion criteria: Patients were excluded if they met any of the following criteria: (1) had secondary gout, such as rheumatoid arthritis, septic arthritis, traumatic arthritis, etc.; (2) had gout in the intermittent period or with tophi; (3) were taking the following drugs: diuretics, pyrazinamide, aspirin, etc.; (4) were pregnancy, breast-feeding women; (5) were suffering from cardiovascular and cerebral vascular disease, and severe trauma or had undergone surgery; (6) had severe infections; (7) were suffering from hepatobiliary disease or whose aspartate aminotransferase (AST) and alanine aminotransferase (ALT) were 2 times higher than upper limit of normal; (8) had serum creatinine ( $\mathrm{SCr}$ ) levels greater than the upper limit of normal; (9) had severe chronic gastrointestinal disease; (10) had hematological diseases or endocrine system diseases; (11) were undergoing cancer treatment; (12) were receiving steroid therapy; or (13) were allergic to those known ingredients in the Chuanhu anti-gout mixture.

Elimination criteria: Patients were eliminated if they met any of the following criteria: (1) did not meet the inclusion criteria, or met any of the exclusion criteria but were chosen by mistaken; (2) fit the inclusion/exclusion criteria but did not use the investigational drugs or were without any follow-up records; (3) were of poor compliance, in violation of the program regulations of concomitant medications, especially the drug interposing to the efficacy and safety of the investigational drugs; or (4) were for other reasons ineligible (such as abnormal break-blind cases).

Falling off standards: Patients were considered to fall off the trial if they met any of the following standards: (1) exited from the trial by themselves (poor efficacy, lost or accidental death); (2) were terminated by the investigator during the test (poor compliance, withdrawal of the informed consents, prohibited medications or safety considerations); (3) experienced serious adverse events, other serious illness or specific physiological changes (accidental pregnancy), should not continue to the trial; (4) had incomplete information, which affected the evaluation of the efficacy and safety; or (5) had serious violations of the experiment's regulations.

The study was conducted in accordance with the Declaration of Helsinki, and the protocol was approved by Qingdao University's ethics committee. All patients provided written informed consents.

\section{Randomization and masking}

Patients were randomly assigned to one of the two treatments (Chuanhu anti-gout mixture $250 \mathrm{ml}$ orally daily or Colchicine mimetic agent). Randomi- 
zation was performed by the block randomization method using a random number table generated by SAS. L Wang and Y Li were in charge of the participants' enrollment, and YG Wang was in charge of the participants' assignment to interventions. The study blind was maintained with the participants, doctors and nurses from the random number assignment, including the test drug number, collection of the data and evaluation, and data management, until the statistical analysis.

\section{Medicine}

The Chuanhu anti-gout mixture was supplied by the Affiliated Hospital of Medical College Qingdao University [12]. The fingerprint and quality standards showed that the main ingredients were resveratrol, emodin, chlorogenic acid, dioscin, etc. (Supplementary Material: Figure S1).

\section{Procedures}

Following screening, eligible patients at the Affiliated Hospital of Medical College Qingdao University were randomized (1:1) to the following two groups: 1) the Chuanhu group, to receive the Chuanhu anti-gout mixture $250 \mathrm{ml}$ orally daily and placebo (mimetic agent of colchicine); or 2) the Colchicine group, to receive Colchicine 1 piece orally ( 0.5 $\mathrm{mg}$ per piece), 2 times daily, for 3 days, later changed to once daily, and placebo (mimetic agent of the Chuanhu anti-gout mixture). All of the patients were treated with etoricoxib $60 \mathrm{mg}$ once daily orally to relieve pain in the affected joints for 10 days.

In addition, all the patients 1) were required to rest remaining in bed and avoiding lifting the affected limb, and avoid overtiring, tension and cold, 2) were given a low purine diet, abstaining from smoking, wine, animal giblets, fish, shrimp and unfermented soybean foods, 3) were required to drink enough water, 2000-3000 $\mathrm{ml}$ daily, 4) received sodium bicarbonate $1.0 \mathrm{~g}$ three times daily orally to alkalinize the urine, and 5) received etoricoxib $60 \mathrm{mg}$ daily orally for 10 days to relieve pain in the affected joints.

The primary outcome was defined as the recurrence rate within three months of patients who had received 10 days of treatment. Secondary outcomes were the changes in white blood cells (WBC) and C-reactive protein (CRP) from the baseline to the follow-up. The adverse reactions of pain, swelling, and activity limitation of the joints (measured on a 1-4 point scale, for absent, mild, moderate, or severe, respectively) were recorded in the patient diaries, and biochemical indicators were detected.

\section{Statistical analysis}

To test whether the Chuanhu anti-gout mixture $250 \mathrm{ml}$ orally daily was inferior to Colchicine for the treatment of acute gouty arthritis, a pre-specified non-inferiority margin of $15 \%$ was used [13]. A sample size of 77 participants per treatment group was needed to reach a power of $80 \%$ and a one-sided type I error of $5 \%$ [14].

The unilateral $U$ test was used to compare the recurrence rates between the two groups after 3 months, and the non-inferiority test results were verified by taking the $95 \%$ confidence interval. For the measurement data, a confidence interval approach was used on an analysis of t-test model, with a one-side $5 \%$ level of significance. For the count data, a confidence interval approach was used on an analysis of $x^{2}$ model, with a one-side $5 \%$ level of significance. And for ranked data, the Kruskal-Wallis test was chosen. For the stability of the results, the per-protocol analysis (PP) and the intention-to-treat analysis (ITT) were both used in the study.

\section{Role of the funding source}

The study was supported by the Affiliated Hospital of Qingdao University Medical College. The sponsor of the study had a role in the study design, but not in data collection, data analysis and data interpretation, or writing of the report. The corresponding author had full access to all the data in the study and had the final responsibility to submit it for publication.

\section{Results}

Between September 1, 2011, and September 30, 2012, 176 patients at the Affiliated Hospital of Qingdao University Medical College were enrolled and randomly assigned to treatment with the Chuanhu anti-gout mixture or with Colchicine. Patient and disease characteristics were similar between the groups (Table 1). One subject in the $\mathrm{CH}$ group withdrew from the trial due to an increased frequency of stool, one was unable to tolerate the bitter taste, and one was removed because the blind was unexpectedly broken, thus the final eligible sample was 85; in the Col group, six patients withdrew for severe diarrhea, two patients withdrew for liver damage, and one patient was excluded for poor compliance and had no follow-up record, and the final eligible sample size was 79 (Figure 1).

In the intent-to-treat (ITT) analysis, the difference of recurrence rate was $-2.22 \% \quad(95 \% \mathrm{CI}$ : $-10.78 \%-6.23 \%)$, while in the per-protocol (PP) analysis, the difference was $-3.51 \%$ (95\% CI: $-12.61 \%, 5.59 \%)$ (Table 2). The 95\% interval confidences were included in the non-inferiority interval $[-15 \%, 15 \%]$; therefore, based on the recurrence rate, the treatment with the Chuanhu anti-gout mixture was not inferior to treatment with Colchicine. 


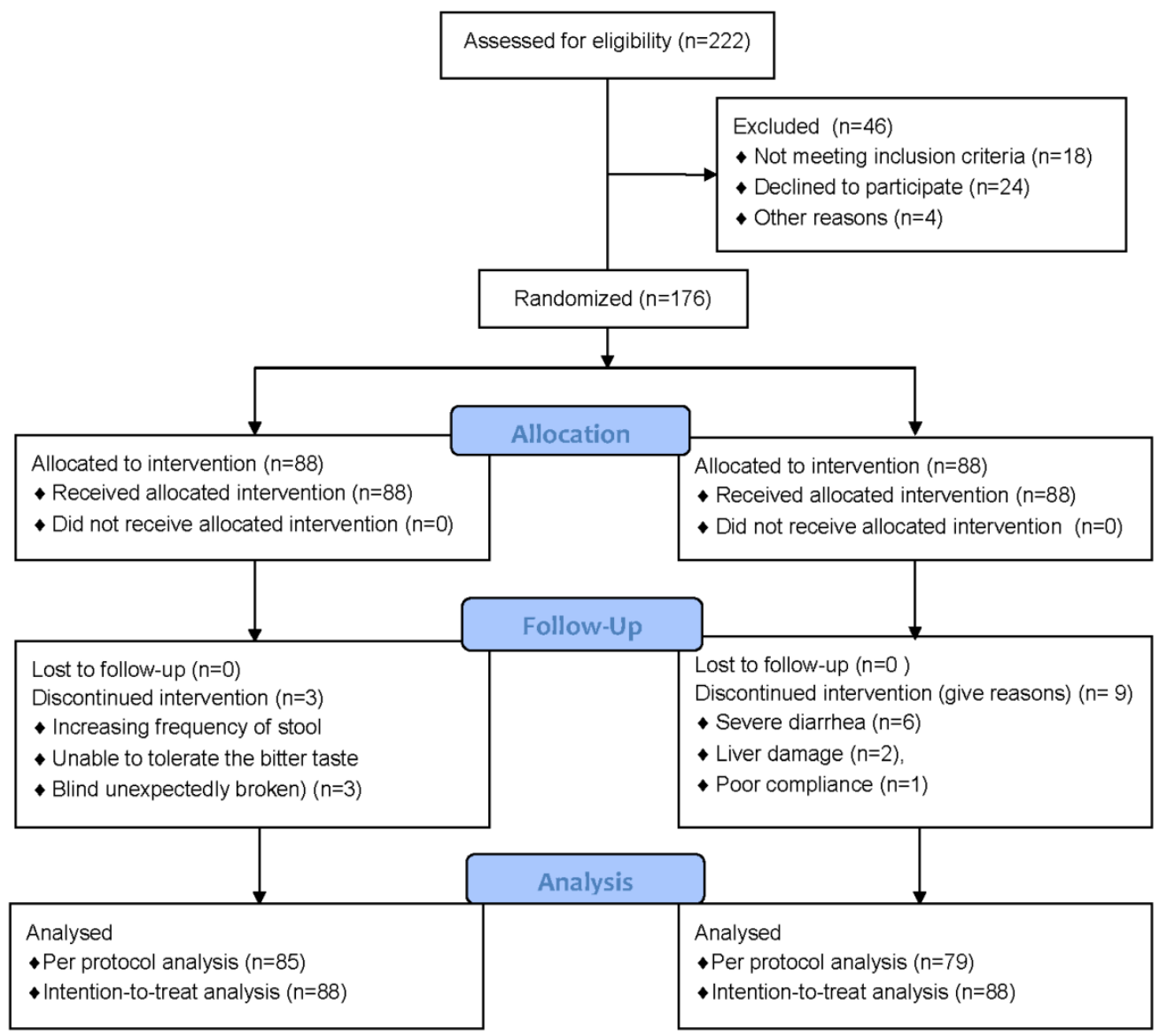

Figure I. Trial profile.

Table I. Patient and disease characteristics at baseline.

\begin{tabular}{cll}
\hline & CH group $(\mathrm{n}=88)$ & Col group $(\mathrm{n}=88)$ \\
\hline Age (years) & $51.76 \pm 13.21$ & $53.82 \pm 14.19$ \\
Women $(\%)$ & $6(6.82 \%)$ & $4(4.55 \%)$ \\
Height $(\mathrm{cm})$ & $1.73 \pm 0.06$ & $1.73 \pm 0.06$ \\
Weight $(\mathrm{kg})$ & $81.40 \pm 12.02$ & $78.82 \pm 11.699$ \\
BMI $\left(\mathrm{kg} / \mathrm{m}^{2}\right)$ & $27.05 \pm 3.21$ & $26.42 \pm 2.92$ \\
Systolic blood pressure $(\mathrm{mmHg})$ & $140.55 \pm 18.01$ & $141.44 \pm 18.36$ \\
Diastolic blood pressure $(\mathrm{mmHg})$ & $88.03 \pm 12.93$ & $86.38 \pm 12.03$ \\
White blood cell count $\left(10^{9} / \mathrm{L}\right)$ & $9.13 \pm 2.91$ & $9.34 \pm 2.58$ \\
C-reactive protein $(\mathrm{mg} / \mathrm{L})$ & $20.44 \pm 16.29$ & $20.48 \pm 13.05$ \\
AST & $26.92 \pm 14.84$ & $22.92 \pm 7.07$ \\
ALT & $32.37 \pm 23.99$ & $22.71 \pm 7.53$ \\
BUN & $5.61 \pm 2.12$ & $5.59 \pm 2.05$ \\
BCr & $83.83 \pm 17.96$ & $82.40 \pm 13.45$ \\
GLU & $6.05 \pm 1.45$ & $5.99 \pm 1.01$ \\
TG & $2.46 \pm 1.57$ & $2.66 \pm 1.82$ \\
TC & $5.56 \pm 1.74$ & $5.67 \pm 1.74$ \\
UA & $465.22 \pm 79.88$ & $449.81 \pm 68.16$ \\
The score of joint pain & $2(1 \sim 2)$ & $2(1 \sim 2)$ \\
The score of joint swelling & $1(0 \sim 2)$ & $1(0 \sim 2)$ \\
The score of limitation of joint activity & $1(1 \sim 2)$ & $1(1 \sim 2)$ \\
\hline *Continuous variables are expressed as the mean \pm standard deviation, grade \\
variables as median (interquartile range) and categorical variables as $\mathrm{n}(\%)$.
\end{tabular}

Differences in the white blood cell count (WBC) and C-reactive protein (CRP) in these two groups, at the baseline and after the follow-up, were consistent with the normal distribution. A one-sided $t$ test was used with a preset non-inferiority test value of $-0.5 \times$ $10^{9} /$ L. The results showed the differences of the means were $0.12 \times 10^{9} / \mathrm{L}\left(95 \%\right.$ CI: $-0.32 \times 10^{9} / \mathrm{L}$ $\left.\sim 0.42 \times 10^{9} / \mathrm{L}\right)$ and $0.09 \times 10^{9} / \mathrm{L}\left(95 \% \mathrm{CI}:-0.29 \times 10^{9} /\right.$ $\left.\mathrm{L} \sim 0.37 \times 10^{9} / \mathrm{L}\right)$, in the PP and the ITT analysis, respectively (Table 3 ). The lower limits of the $95 \%$ confidence interval of WBC in the two groups were both greater than the preset non-inferiority test value of $-0.5 \times 10^{9} / \mathrm{L}$, thus the non-inferiority test was qualified.

Table 2. The recurrence rates (RR) in the $\mathrm{CH}$ and $\mathrm{Col}$ groups by the non-inferiority test.

\begin{tabular}{|c|c|c|c|c|c|c|c|}
\hline & Group & & Recurrence No. & $\begin{array}{l}\mathrm{RR} \\
(\%)\end{array}$ & $\begin{array}{l}\text { Rate difference (\%) (95\% } \\
\text { confidence interval) }\end{array}$ & $u$ & $\begin{array}{l}P \\
\text { value }\end{array}$ \\
\hline \multirow[t]{2}{*}{ PP } & $\mathrm{CH}$ & 85 & & 12.94 & $-3.51(-12.61,5359)$ & 2.06 & 0.020 \\
\hline & $\mathrm{Col}$ & 79 & & 16.45 & & & \\
\hline \multirow[t]{2}{*}{ ITT } & $\Gamma \mathrm{CH}$ & 88 & & 12.50 & $-2.22(-10.78 \%, 6.23 \%)$ & 2.45 & 0.007 \\
\hline & Col & 88 & 13 & 14.77 & & & \\
\hline
\end{tabular}

As the data of the C-reactive protein (CRP) could not be assumed to have a normal distribution, the Kruskal-Wallis rank sum test was used with a preset non-inferiority test critical value of -1 . Mediate differences were $-0.25 \mathrm{mg} / \mathrm{L}$ (95\% CI: $-0.39 \mathrm{mg} / \mathrm{L} \sim 0.36$ 
$\mathrm{mg} / \mathrm{L}$ ) and $-0.18 \mathrm{mg} / \mathrm{L}$ (95\% CI: $-0.34 \mathrm{mg} / \mathrm{L} \sim 0.31$ $\mathrm{mg} / \mathrm{L}$ ), in the PP and the ITT analysis, respectively (Table 3).The lower limits of the 95\% confidence interval were greater than the default $-1.0 \mathrm{mg} / \mathrm{L}$, thus the non-inferiority test was eligible.

The decrease of pain score in the affected joint was larger in $\mathrm{CH}$ group than in Col group (mean rank 97.44 vs $79.56, P=0.013$ ), while the decrease of swelling and limitation scores in the two groups had no significant differences $(P>0.05)$ (see Supplemental Table 1). There were no cases of death, gastrointestinal bleeding, and kidney function damage in the two groups. The subjects withdrew from the trial if any adverse reactions occurred, and their data were not included in the efficiency analysis. There were 25 cases with adverse reactions in the Col group, while in the $\mathrm{CH}$ group, 2 patients withdrew the study (one medicine bitter taste, another for an adverse reaction) (Table 4). The rates of total adverse reactions were $3.27 \%$ and $28.41 \%$ in $\mathrm{CH}$ group and $\mathrm{Col}$ group, respectively, with an odds ratio (OR) of 0.06 (95\% CI: $0.01 \sim 0.26)$.

Table 3. Non-inferiority test of WBC and CRP changes before and after treatments in the $\mathrm{CH}$ and Col groups $\left(\times 10^{9} / \mathrm{L}\right)$.

\begin{tabular}{lllllll}
\hline & Group & $\mathrm{n}$ & \multicolumn{2}{l}{ WBC } & & $\mathrm{CRP}$ \\
\cline { 3 - 7 } & & & $\overline{\mathrm{X}}(S)$ & $P$ value & $\mathrm{M}(\mathrm{iQR})^{*}$ & $P$ value \\
\hline PP & $\mathrm{CH}$ & 85 & $2.20(2.48)$ & 0.003 & $12.40(3.61)$ & 0.003 \\
& $\mathrm{Col}$ & 79 & $2.08(2.38)$ & & $12.65(4.87)$ & \\
\multirow{2}{*}{ ITT } & $\mathrm{CH}$ & 88 & $2.20(2.46)$ & $<0.001$ & $12.41(5.62)$ & $<0.001$ \\
& Col & 88 & $2.11(1.95)$ & & $12.59(4.34)$ & \\
\hline
\end{tabular}

${ }^{*} \mathrm{M}(\mathrm{iQR})$ : Median (interquartile range).

Table 4. Rates of Adverse reaction Occurrence in the $\mathrm{CH}$ and Col Groups.

\begin{tabular}{llllll}
\hline & $\mathrm{CH}(\mathrm{n}=88)$ & $\mathrm{Col}(\mathrm{n}=88)$ & $\mathrm{OR}(95 \% \mathrm{CI})$ & $\mathrm{X}^{2}$ & P value \\
\hline total & $2(2.27 \%)$ & $25(28.41 \%)$ & $0.06(0.01 \sim 0.26)$ & 23.14 & $<0.001$ \\
diarrhea & $1(1.14 \%)$ & $21(23.86 \%)$ & $0.04(0.01 \sim 0.28)$ & 20.78 & $<0.001$ \\
nausea & $1(1.14 \%)$ & $3(4.92 \%)$ & $0.33(0.03 \sim 3.19)$ & 1.023 & 0.621 \\
vomiting & 0 & $1(16.39 \%)$ & - & 1.006 & 1.000 \\
\hline
\end{tabular}

Table 5. Biochemical markers changes before and after treatment in the $\mathrm{CH}$ and $\mathrm{Col}$ Groups.

\begin{tabular}{lllll}
\hline & $\mathrm{CH}$ & $\mathrm{Col}$ & $\mathrm{t}$ value & P value \\
\hline AST & $6.36 \pm 14.66$ & $-4.42 \pm 10.87$ & -5.32 & $<0.001$ \\
ALT & $12.92 \pm 23.67$ & $-6.17 \pm 13.92$ & -6.24 & $<0.001$ \\
$\mathrm{BUN}$ & $-0.97 \pm 9.96$ & $-0.90 \pm 9.92$ & 0.04 & 0.966 \\
$\mathrm{Cr}$ & $5.88 \pm 16.13$ & $-8.88 \pm 16.11$ & -5.86 & $<0.001$ \\
GLU & $0.33 \pm 1.54$ & $0.08 \pm 1.13$ & 1.18 & 0.24 \\
TG & $0.59 \pm 1.00$ & $0.51 \pm 1.59$ & -0.4 & 0.69 \\
TC & $0.60 \pm 1.51$ & $0.50 \pm 1.96$ & -0.38 & 0.708 \\
UA & $63.17 \pm 42.06$ & $29.00 \pm 43.38$ & 5.12 & $<0.001$ \\
AST=aspartate aminotransferase, ALT=alanine aminotransferase, BUN=blood urea \\
nitrogen, Cr=creatinine, GLU=blood sugar, TG=triglycerides, TC=total cholesterol, \\
UA=blood uric acid.
\end{tabular}

The levels of uric acid, alanine aminotransferase, aspartate aminotransferase and creatinine were decreased more significantly in the $\mathrm{CH}$ group than in the Col group $(P<0.05)$, while the differences of other indicators between the two groups were not significant $(P>0.05)$ (Table 5).

\section{Discussion}

This present study showed that in patients with acute gouty arthritis, the Chuanhu anti-gout mixture was non-inferior to Colchicine in lowering the recurrence rate (treatment difference within the prespecified non-inferiority margin of $15 \%$ ), white blood cell count (treatment difference within the prespecified non-inferiority margin of $-0.5 \times 10^{9} / \mathrm{L}$ ), and C-reactive protein (treatment difference within the prespecified non-inferiority margin of $-1.0 \mathrm{mg} / \mathrm{L}$ ). The Chuanhu anti-gout mixture was associated with a better effect in relieving pain in the affected joint, but it was not associated with changes of swelling and limitation scores of the affected joint, or with blood urea nitrogen, blood sugar, triglycerides and total cholesterol, compared to Colchicine. However, the Chuanhu anti-gout mixture was associated with significantly fewer adverse events (especially diarrhea) and lower levels of aspartate aminotransferase, alanine aminotransferase, creatinine and blood uric acid compared to Colchicine.

Colchicine, a highly toxic alkaloid extracted from plants of the genus Colchicum (autumn crocus) has been used to treat gout for 2,000 years [15]. Colchicine is regarded as beneficial in the treatment of acute gout, but it has a high frequency of gastrointestinal adverse events, such as diarrhea, before the relief of gout symptoms $[16,17]$. In addition, the administration of colchicine by the intravenous route has fallen into disrepute with an FDA report recording 33 deaths between 1985 and 1997 [18]. The present study confirmed that there is high frequency of adverse events in gout patients treated with Colchicine, and it showed that treatment with Chuanhu anti-gout mixture, a Chinese Traditional herbal compound, could decrease the incidence of adverse events, mainly diarrhea.

The levels of blood uric acid, aspartate aminotransferase, alanine aminotransferase and creatinine were significantly different between the Chuanhu anti-gout mixture group and the Colchicine group, indicating that the Chuanhu anti-gout mixture may be superior to colchicine in lowering blood uric acid, thus protecting the liver and kidney function.

The possible limitation of this study is the short follow-up period. In 10 days of therapy and 12 weeks of follow-up, the additional recurrence of acute gouty arthritis could not be examined, as well as that of ad- 
verse events, might restrict the expansion of those drugs. In addition, the study was conducted in Qingdao, a coastal city, with drinking and daily consumption of meat and seafood as the main causes of gout [4]. Whether the Chuanhu anti-gout mixture would be effective in other patients needs to be validated in multiple center clinical trials. Nevertheless, some studies have shown that Chinese herbal medicine may have clinical effectiveness for functional recovery in patients with gout and may lead to a safe control of serum uric acid level and inflammation severity [19].

In conclusion, the Chuanhu anti-gout mixture can alleviate acute gouty arthritis symptoms, the clinical efficacy of which was not inferior to colchicine. Meanwhile, it can lower blood uric acid by upregulating the expression of the urate anion transporter protein. No serious adverse reactions were found in this study. In short, the Chuanhu anti-gout mixture provides new ideas and methods for the treatment of acute gouty arthritis.

\section{Conclusion}

This trial showed that from an effectiveness point of view, the Chuanhu anti-gout mixture could be the superior choice for the treatment of acute gouty arthritis, for its efficacy (non-inferior to colchicine), lower incidence of adverse events and protection of kidney and renal function (superior to colchicine). The mechanisms of the Chuanhu anti-gout mixture in reducing the recurrence rate of acute gouty arthritis and in reducing the levels of uric acid, aspartate aminotransferase, alanine aminotransferase and creatinine need further investigations.

\section{Supplementary Material}

Figure S1. The TCM fingerprint of the known active ingredients of the Chuanhu anti-gout mixture.

http://www.medsci.org/v11p0880s1.pdf

\section{Competing Interests}

The authors declare that they have no competing interests.

\section{Acknowledgement}

Thank you to all the participants in this clinical trial, including the patients, doctors and nurses. Youxin Wang was supported by the Beijing Higher Education Young Elite Teacher Project (YETP1671) and the Beijing Nova Program (Z141107001814058).

\section{References}

1. Roddy E, Zhang $W$ and Doherty $M$. The changing epidemiology of gout. Nat Clin Pract Rheumatol. 2007; 3: 443-9.

2. Rho YH, Zhu Y, and Choi HK. The Epidemiology of Uric Acid and Fructose. Semin Nephrol. 2011; 31: 410-9.
3. Richette P, Bardin T Gout Lancet. 2010; 375: 318-28.

4. Miao Z, Li C, Chen Y, et al. Dietary and lifestyle changes associated with high prevalence of hyperuricemia and gout in the Shandong coastal cities of Eastern China. J Rheumatol. 2008; 35: 1859-64.

5. Annemans L, Spaepen E, Gaskin M, et al. Gout in the UK and Germany: prevalence, comorbidities and management in general practice 2000-2005. Ann Rheu Dis 2008; 67: 960-6.

6. Primatesta P, Plana E, Rothenbacher D. Gout treatment and comorbidities: a retrospective cohort study in a large US managed care population. BMC Musculoskelet Disord. 2011; 12: 103.

7. Choi HK, De Vera MA, Krishnan E. Gout and the risk of type 2 diabetes among men with a high cardiovascular risk profile. Rheumatology (Oxford). 2008; 47: 1567-70.

8. Choi HK, Ford ES, Li C Curhan G. Prevalence of the metabolic syndrome in patients with gout: the Third National Health and Nutrition Examination Survey. Arthritis Rheum. 2007; 57: 109-15.

9. Kim SY, Guevara JP, Kim KM, et al. Hyperuricemia and coronary heart disease: a systematic review and meta-analysis. Arthritis Care Res 2007; 62: $170-80$

10. $\mathrm{Li} \mathrm{T}, \mathrm{Chen} \mathrm{SL}$, Dai Q, et al. Etoricoxib versus indometacin in the treatment of Chinese patients with acute gouty arthritis: a randomized double-blind trial. Chin Med J (Engl). 2013; 126: 1867-71.

11. Ghosh P, Cho M, Rawat G, Simkin PA, Gardner GC. The treatment of acute gouty arthritis in complex hospitalized patients with anakinra. Arthritis Care Res. 2013; 65: 1381-4.

12. Wang J, Yang $\mathrm{X}$, Zhang C, Wu A. Determination of Emodin in Chuanhu Tongfeng Mixture by HPLC. China Pharmaceuticals. 2012; 21: 55-56.

13. Committee for Medicinal Products for Human Use, Efficacy Working Party, Committee for Release for Consultation, Committee for Medicinal Products for Human Use (CHMP). Guideline on the choice of the non-inferiority margin. Stat Med. 2006; 10: 1628-38.

14. Blackwelder WC. Proving the null hypothesis in clinical trials. Control Clin Trials. 1982; 3: 345-53.

15. Grahame R. Is there still a place for colchicine in the treatment of acute gout? Int J Clin Prac. 2007; 61: 1966-7.

16. Rott KT, Agudelo CA. Gout. J Am Med Assoc. 2003; 289: 2857-60.

17. Schlesinger N, Schumacher R, Catton M, Maxwell L. Colchicine for acute gout. Cochrane Database Syst Rev. 2006; 18: CD006190.

18. Bonnel RA, Villalba ML, Karwoski CB, Beitz J. Deaths associated with inappropriate intravenous colchicine administration. J Emerg Med. 2002; 22: 385-7.

19. Li XX, Han M, Wang YY, Liu JP. Chinese herbal medicine for gout: a systematic review of randomized clinical trials. Clin Rheumatol. 2013; 32: 943-59. 